\title{
A Practical Guide to Quality Improvement in Nuclear Medicine*
}

\author{
Mary Beth Farrell, $\mathrm{CNMT}^{1}$ and Sue H. Abreu, $\mathrm{MD}^{2}$ \\ ${ }^{1}$ Intersocietal Accreditation Commission, Ellicott City, Maryland; and ${ }^{2}$ IAC Board of Directors
}

\begin{abstract}
Innovations and improvements in the field of nuclear medicine have created remarkable image accuracy and detail, which allow physicians to better diagnose disease. This ability has led to dramatic differences in patient care. To ensure that these diagnoses are reliable, imaging facilities must constantly monitor and seek to improve their practices. Quality improvement is a formal process of examining and improving performance through the analysis of data with the primary goal of enhancing patient care. Quality improvement activities in a nuclear medicine laboratory should emphasize accuracy and efficiency, patient and staff safety, and the patient's experience during care. Quality improvement in the nuclear medicine laboratory can potentially reduce the number of studies that need to be repeated because of poor quality, increase diagnostic accuracy, reduce radiation exposure, increase patient satisfaction, and save resources. This article will review the process of quality improvement; provide detailed, step-by-step instructions with special emphasis on project selection and data collection; and show examples of how to perform quality improvement projects.
\end{abstract}

Key Words: quality improvement; quality control; accuracy; efficiency

J Nucl Med Technol 2012; 40:211-219

DOI: 10.2967/jnmt.112.111880

\section{$\mathbf{S}$} novations and improvements in nuclear medicine have been nothing short of revolutionary. The remarkable image accuracy and detail allow physicians to diagnose disease without the more invasive procedures used in the past. This ability has led to dramatic differences in patient care. To be certain that these diagnoses are reliable, imaging facilities must constantly monitor and seek to improve their practices. To ensure that images are indeed accurate and of high quality, the ongoing assessment of laboratory performance is critical (1).

\footnotetext{
Received Jul. 29, 2012; revision accepted Sep. 18, 2012.

For correspondence or reprints contact: Mary Beth Farrell, CNMT, 27 Boxwood Lane, Langhorne, PA 19047.

E-mail: farrell@intersocietal.org

Published online Oct. 15, 2012.

*NOTE: FOR CE CREDIT, YOU CAN ACCESS THIS ACTIVITY THROUGH THE SNMMI WEB SITE (http://www.snmmi.org/ce_online) THROUGH DECEMBER 2014.

COPYRIGHT (C) 2012 by the Society of Nuclear Medicine and Molecular Imaging, Inc.
}

Numerous stakeholders, including insurance payers, referring physicians, and patients, are concerned about the quality of imaging. Therefore, laboratories must document the quality measures and processes that ensure their study results are valid, not only to provide superior care but also to promote the overall success and viability of the laboratory.

Quality improvement is a formal process to examine and improve performance through the analysis of data (2). The primary goal of quality improvement is to enhance patient care. Quality improvement activities in a nuclear medicine laboratory should emphasize the accuracy and efficiency of patient care, patient and staff safety, and the patient's experience during care. Projects should focus on the human factors that can lead to variations in care, not to blame someone, but to discover the changes that will most effectively improve outcomes.

This article will review the process of quality improvement for a nuclear medicine laboratory, provide detailed step-by-step instructions, and give examples of how to perform quality improvement projects.

\section{MONITORING VERSUS IMPROVING: QUALITY CONTROL VERSUS QUALITY IMPROVEMENT}

Every nuclear medicine laboratory monitors many aspects of its practice. Quality control, one type of monitoring, refers to the procedures and tests performed on equipment to ensure proper function. $\gamma$-camera daily uniformity tests, weekly resolution/linearity tests, and monthly center-ofrotation tests are typical quality control measurements. If monitored parameters fall outside the laboratory's acceptable range, the staff intervenes, requesting service for the device that failed to meet quality control standards. Although this does improve practice quality, this is not a quality improvement project. In contrast, a quality improvement project involves a repeating cycle of analysis and implementation of change that leads to sustained performance at a selected level of achievement.

Laboratories may also monitor other aspects of performance such as patient no-show rates, patient satisfaction, or revenue collection rates. These performance aspects are important for the laboratory to measure and track but are not quality control. By watching these parameters for change, the laboratory can react before problems compromise patient care, just as it can react to small variations in quality control 
results before there is complete failure of a device. Again, this is monitoring rather than performing a quality improvement project.

Through monitoring, the laboratory may discover processes that need systematic improvement. When the staff identifies an area for improvement, the problem-solving process begins. This is the initial phase of quality improvement. The team-based problem-analysis process can lead to significant advances in patient care, patient safety, and practice efficiency. Quality control, other types of performance monitoring, and quality improvement are all critical to maintaining overall practice quality.

\section{APPROACHING QUALITY IMPROVEMENT: THE PLAN-DO-STUDY-ACT (P-D-S-A) CYCLE}

A planned, structured problem-solving process, as opposed to haphazard, random attempts to solve problems, produces better long-term solutions. Problem-solving processes should use data to measure performance so that the staff can objectively observe change (3). Quality improvement is the process of examining data and making changes systematically to improve performance.

Organized data collection and evaluation result in more meaningful system changes than do knee-jerk reactions and policing. To treat just the symptoms will not create longterm results. The long-term cure, the solution to problems, is more valuable. For example, the systematic evaluation of a significant sample of false-positive or false-negative studies will result in more appropriate and significant improvements than will discussions of isolated misdiagnoses.

To conduct a full quality improvement project, the staff needs a problem-solving model. One of the most frequently

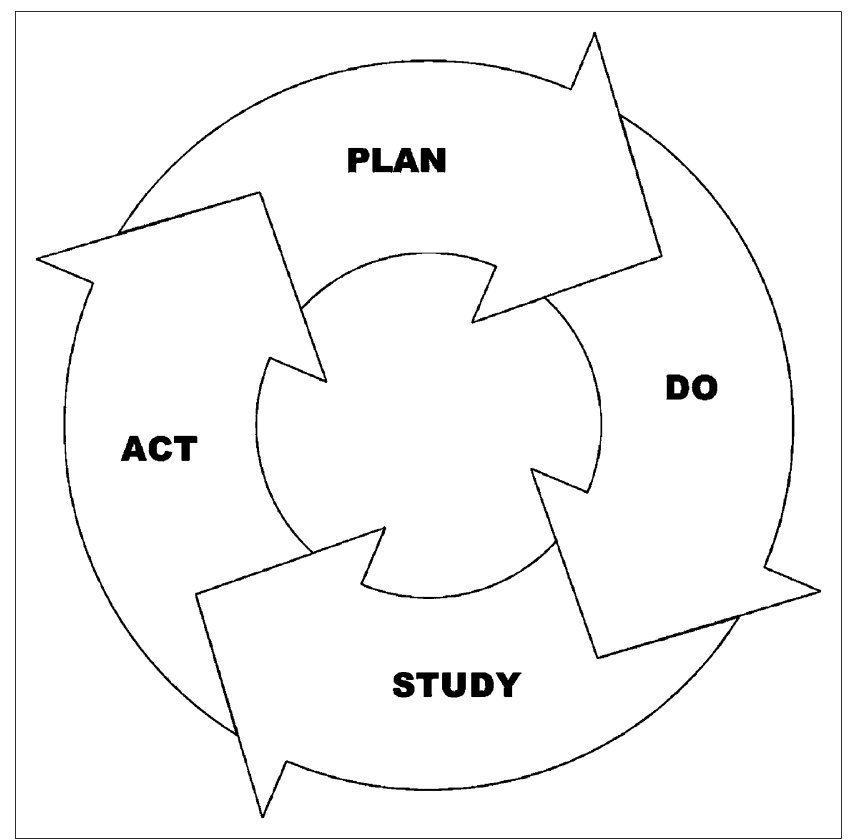

FIGURE 1. P-D-S-A Cycle: A problem-solving model for effecting change. used and simplest frameworks is the P-D-S-A cycle for affecting change (Fig. 1) (4).

- Plan: An area for improvement is identified and a plan is developed.

- Do: Measurements are performed and analyzed.

- Study: Results are summarized and evaluated.

- Act: Possible interventions are assessed and changes implemented.

In the "plan" stage, the staff selects the problem, issue, or area for improvement and clearly states the objective. For example, an overall goal can be to increase the compliance of nuclear cardiology reports with published guidelines. The laboratory also makes predictions or sets thresholds, such as "greater than $90 \%$ of final reports will contain all the required reporting elements."

Next, the staff selects what to measure, who to collect the measurements, when to collect the measurements, and what collection tools to use. To gather the data, the laboratory could use a simple checklist of required reporting elements to review 5\% of its annual patient volume. The KISS principle- "Keep It Simple, Stupid"-is important to keep in mind. The simpler the data collection method, the less stress the improvement project will cause to the staff involved.

During the "do" stage, the staff collects and analyzes the data. The laboratory makes observations and documents what occurs. Data evaluation can be as simple as finding the percentage of results within the acceptable range. To perform small projects to improve quality in nuclear medicine, the facility does not need advanced statistical analyses.

In the "study" stage, the staff scrutinizes the results compared with the predictions or thresholds and thoroughly evaluates data outliers to find possible causes and solutions.

The staff next summarizes what the data analysis revealed. In the nuclear cardiology reporting example, the laboratory determines that $40 \%$ of reports do not comply with requirements because they lack the exact dose of the administered radiopharmaceutical. Further investigation reveals that the physician dictated the prescribed dose rather than the administered dose when the technologist did not document the exact dose on the worksheet.

The last stage is to "act." The laboratory decides what intervention will help and when to implement the change. Again, using the reporting example, they could update the patient worksheet to include instructions that the physician must report the administered radiopharmaceutical dose to 1 decimal point. Alternatively, the laboratory could revise the worksheet to make space for a sticker with the dose calibrator measurement.

After the laboratory makes the selected changes, it recollects data and evaluates the results. If the data demonstrate an adequate improvement, then the laboratory incorporates the change into its standard operating procedure. If quality does not improve, then the change is discarded, and the cycle begins again with a new intervention, data collection, and result analysis. The entire P-D-S-A cycle 


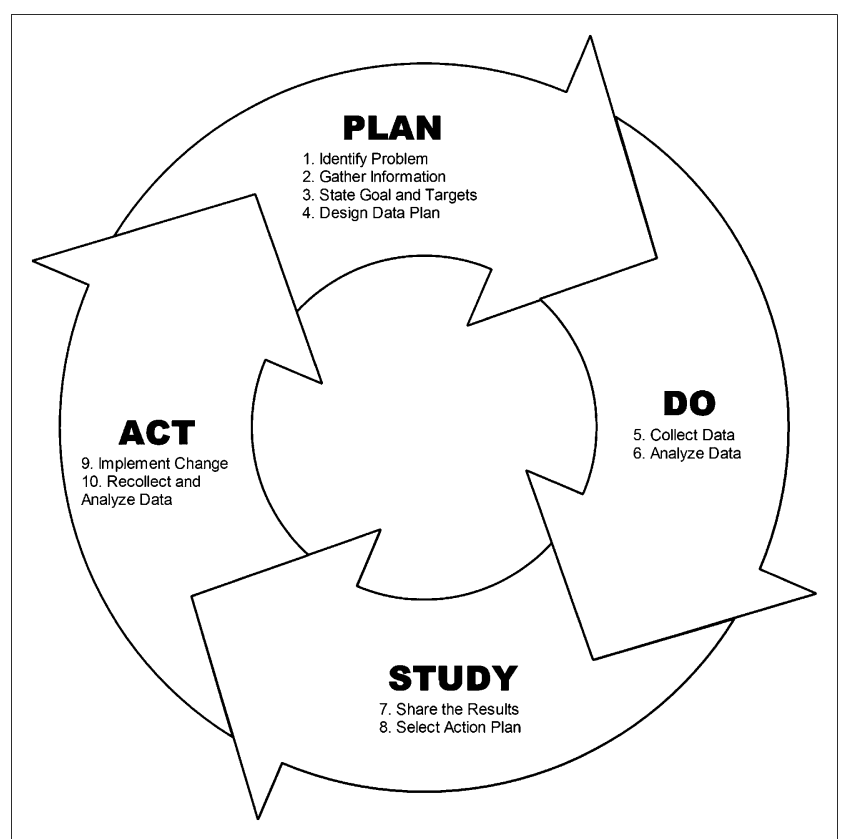

FIGURE 2. P-D-S-A cycle with detailed steps.

is repeated until the desired goal is achieved, as demonstrated in Appendix A (5).

\section{UNDERSTANDING QUALITY IMPROVEMENT: THE TEN STEPS EXPLAINED}

The P-D-S-A cycle is the basis for all quality improvement projects. The cycle can be broken down into detailed steps to aid understanding (Fig. 2). The "plan" stage is divided into 4 steps, and the "do," "study," and "act" stages are each divided into 2 steps.

\section{Step 1: Identify a Potential Problem or Area of Concern}

The person responsible for oversight of the laboratory's quality improvement program should not choose quality improvement projects in isolation. Everyone in the department should be involved in the discussion of potential projects and questioned about problems in the department.

The problems could relate to any aspect of patient care or department function. This is a brainstorming session. Sources of potential quality improvement projects include accreditation survey or licensing inspection reports; new regulations, guidelines, or standards; news stories about adverse events at other facilities; and medical journal articles. To help the staff think of ideas, reviewing the concepts of high risk, high volume, and high cost may be helpful.

High-risk areas are important because just a single highrisk event may have devastating consequences. For example, although uncommon, a patient undergoing an exercise stress test could have a serious cardiac event and die if staff reaction is slow. Therefore, having a strong emergency response plan is essential. Because cardiac events are so rare, staff can easily forget to check all the equipment and supplies necessary for a rapid, effective resuscitation.

A useful monitoring process would determine compliance with all the emergency response-related policies of the facility. For example, a checklist could include the following: Is the defibrillator tested on schedule? Are all emergency response medications present and unexpired? Is the oxygen tank full? Are oxygen delivery devices readily available? Do all personnel know their duties in such an emergency? If monitoring these areas reveals any deficiencies, a quality improvement project to ensure departmental compliance with all emergency response policies may prevent a patient's death.

High-volume procedures are beneficial areas for quality improvement projects because small changes can affect a large proportion of the practice. Because myocardial perfusion imaging studies are a large portion of most practices, a project that improves their quality will benefit many patients. For example, the laboratory could evaluate the adequacy of patient preparation before pharmacologic stress testing. Alternatively, the laboratory could compare the results of myocardial perfusion studies with cardiac catheterization results to determine whether the rate of false-positive studies is comparable to those of other practices. If these evaluations reveal a deficiency, the quality improvement process can be used to improve the studies.

High-cost procedures are valuable targets for quality improvement projects because cost efficiency is essential, especially in this era of reduced reimbursement. The cost of radiopharmaceuticals is a significant part of the budget of any nuclear medicine department. A nuclear medicine department that has a large number of patients who do not arrive for their appointments can be plagued with wasted doses. If the clinic monitors its no-show rate and finds a problem, a quality improvement project that analyzes the reasons for no-shows and reviews the scheduling and confirmation process for appointments will undoubtedly uncover ways to decrease the number of missed appointments, thus saving the laboratory considerable money and frustration.

When the laboratory has a broad range of ideas, these ideas can be consolidated and prioritized. Again, the concepts of high risk, high volume, and high cost are helpful when prioritizing the potential projects. Once it selects the most significant quality improvement projects, management must then ensure that there are sufficient resources available to conduct the projects-especially, sufficient time for staff to do this extra work without compromising other department responsibilities. Management should appoint a quality improvement project leader to guide the project. In a larger facility, the leader may head a small team that oversees the project.

\section{Step 2: Gather Information to Understand the Full Extent of the Problem}

As the team studies the problem in depth, they should remember 2 key principles: the $85 / 15$ principle and the $80 / 20$ principle. 
The 85/15 principle maintains that usually processes, not people, are the cause of problems. This rule expresses that $85 \%$ of the time the process, structure, or practices of the organization are the root cause of the problem or error. Only about $15 \%$ of the time are people the cause (6). As an example, if a nuclear medicine department frequently runs out of clean laundry, the reason may be that the technologist occasionally forgets to order the laundry. However, if the hospital has a system that allows staff to order laundry only once a week during a specific time, the cause of the problem may be that the system is too inflexible to meet the needs of the department. The $85 / 15$ principle is a reminder to focus on processes rather than people.

The $80 / 20$ principle states that $80 \%$ of the problems or errors are the result of only $20 \%$ of the factors. More simply expressed, a small proportion of things causes most of the problems. This principle underlines the importance of focusing on the " $20 \%$ that matters" to manage those things that really make a difference.

As the quality improvement team analyzes the problem to determine potential solutions, the team may need input from many sources. A wide range of relevant factors should be explored, until the team has an in-depth understanding of the problem and can move to step 3 .

\section{Step 3: State the Goal and Set Targets}

Based on the research and analysis, the team establishes a goal for the project. The goal can be a relatively broad statement. For example, the broad goal could be "to reduce the number of inappropriate studies performed."

The goal must be measurable. The team must quantify the target so that the impact of interventions can be judged objectively. For a larger project, the team may establish intermediate targets. Over time, the team may change the goal as new data are found or practical limitations discovered.

A benchmark based on the medical literature, multipractice survey data, or other community standards is the best target. For instance, a laboratory could set its initial threshold for appropriateness of myocardial perfusion studies at $80 \%$ on the basis of the published literature (7). Because such benchmarks are not always available, the target may also be a measurable goal selected by the team on the basis of experience.

When benchmarks are used, a key decision is whether to strive for the minimum acceptable performance, the community average, or a higher standard. One approach is to create stepwise targets that move the laboratory first to the minimums and then gradually to the top. Quality improvement projects may extend over several months or years, depending on the complexity of the project. There is no set timetable; steady progress toward the established target is expected. To find solutions that create sustainable improvement is more important than to quickly reach a target but then to lose that level of performance.

Once the target is established, the quality improvement team must determine what data to gather.

\section{Step 4: Design a Data Collection Strategy}

Data collection is absolutely essential. Proper data collection requires that measurements be accurate. The adage "garbage in, garbage out" could not be truer than in quality improvement. If the collected data are unreliable, the team will learn nothing substantial. In addition, measurements must be selective and meaningful. For example, a laboratory will not be improving its practice if it decides to conduct a quality improvement project concerning misadministrations of radioactive material when it already knows there have been no misadministrations in several years. Remember, monitoring is important and necessary but is not the same as quality improvement.

Sample Size: How Many Cases to Study? Perhaps the most frequent question regarding data collection is, "How many patients do I have to measure?" or "What size sample do I need?" The "sample" is the part of the population from which to gather the needed information. In other words, the sample is the number of observations to be made or the number of patients to be studied. A too-small sample will not produce useful results because the data might miss significant problems or an occasional random event could skew the results. A sample must be large enough to represent the study population, find important error trends, and ensure that any difference found is truly significant. However, no one wants to expend time and resources collecting more data than necessary (8).

Using complicated statistical calculations is usually not necessary to determine sample size for nuclear medicine quality improvement studies. Remember the KISS principle. As a general rule of thumb, review of 30-55 patients is acceptable. To learn more about calculating sample size, the U.S. Health Resources and Services Administration provides a straightforward explanation on its Web site (9).

The size of the sample needed to reveal significant problems depends on how much error is tolerable. To evaluate a process for which no error is acceptable, a $100 \%$ sample is necessary. That is, the review would have to include all events to ensure that no error occurred. In a laboratory that performs only occasional radionuclide therapies (e.g., fewer than 50 per year), this sample size would be appropriate and reasonable.

In contrast, at a busy clinic that annually performs several hundred to several thousand imaging procedures, a sample of 39 studies per year could detect a defect rate of $10 \%$. In other words, when the laboratory can tolerate errors in up to $10 \%$ of cases, a review of just 39 studies would suffice. If all 39 studies comply with the criteria, then $90 \%$ of all studies are likely in compliance. Similarly, if the laboratory determined that its error tolerance was no more than $5 \%$, then the laboratory would need a sample size of 55 cases. So that a laboratory does not need to calculate the sample size, the Nuclear Regulatory Commission provides tables that show the needed sample size based on the lot size (the total population to study) and the percentage of tolerable defects (10). 
Sampling Method: Which Cases to Study? Another common question is, "How do we select the cases to review?" This question encompasses the issues of data collection frequency (how often) and duration (how long). The team could collect data on every patient, every day, for 2 wk or could randomly select 1 patient per day for $4 \mathrm{mo}$. The method of sampling affects how adequately the data represent the population of patients and the factors that influence the process. There are no strict rules. The laboratory must rely on logic and judgment. For example, if the laboratory is evaluating nuclear cardiology reports and each of 5 doctors reads a specific day every week, the sample would need to encompass all days of the week to get data on all 5 doctors. In contrast, if the doctors rotate on a weekly basis, a sample using studies from only Wednesdays would work, all other factors being equal.

There are many sampling methods available. The 3 most useful for quality improvement projects are consensus sampling, simple random sampling, and proportionalstratified random sampling (11).

Consensus sampling gathers data on every member of the population. When the population is sufficiently small, the entire population may be included, such as in the prior example of occasional radionuclide therapies. Similarly, if a laboratory wants to study the accuracy of gallbladder ejection fractions, and the annual volume of hepatobiliary studies is only 80 patients, to evaluate every study for the year would be feasible.

A simple random sample allows every member of a population to have an equal or defined chance of being included in the sample. For example, if medical record numbers were evenly distributed, a sample using all patients whose record number ends in 5 would give a simple random sample of $10 \%$ of the population. A random-number generator or table may facilitate the selection of the sample.

The proportional-stratified approach to random sampling divides the total population into categories. In a nuclear medicine department, the categories might describe the types of studies performed (nuclear cardiology, general nuclear medicine, or PET). The proportion of patients selected for the sample in each category should match that in the population as a whole. For instance, the annual procedure volume of a nuclear medicine department might be $50 \%$ nuclear cardiology, 30\% general nuclear medicine, and 20\% PET. A proportional-stratified sample will include studies on the basis of these category percentages. So, when the total sample is $\mathbf{5 0}$ studies, 25 studies will be nuclear cardiology, 15 studies will be general nuclear medicine, and 10 studies will be PET.

Once the team has developed the data collection strategy, data collection can begin.

\section{Step 5: Collect Data}

To collect the data, first the team must decide in what format to record the data. The simplest approach is to use a form or checklist and gather the data by hand from sources such as a database, electronic medical record, report, or directly from the patient (Fig. 3). The form should include enough information to allow for data validation, if needed. For example, the medical record number or patient initials and date of study can identify the source of the data. Later in the quality improvement process, the team will investigate outliers. Having a means to identify the source is helpful for deeper analysis.

When necessary, patient identity data may be used for quality improvement; however, the collection tool should gather the least such data needed.

The data collection tools or sheets should include all variables that may be relevant to the problem. To slightly overgather data is generally more efficient than to miskey information and have to go back later to pull more information. On the other hand, the collection of data should not be too laborious. When in doubt apply the KISS principle, keep it simple.

Data analysis follows data collection.

\section{Step 6: Analyze the Data}

Usually, data analysis begins with simple addition and calculation of a percentage of compliance with the criteria. For a multicycle project, a graph showing the trend over time and the target performance level is helpful. For example, when a laboratory measures the percentage of appropriate studies

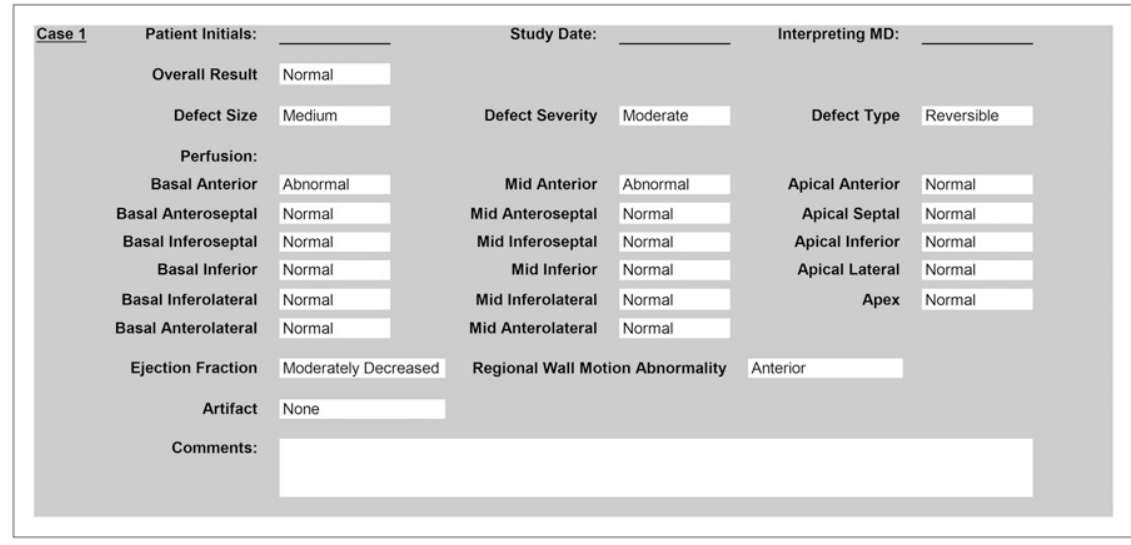

FIGURE 3. Sample data collection tool to assess interobserver variability in reporting of myocardial perfusion defects. Tool documents overall study result (normal/ abnormal); defect size (small/medium/ large), severity (mild/moderate/severe), and type (reversible/mixed/persistent); location based on 17-segment model (normal/ abnormal); ejection fraction (normal/mildly decreased/moderately decreased/severely decreased); regional wall motion abnormality (anterior/lateral/inferior/septal/ apical); and artifact (none/breast tissue/ soft tissue/motion/subdiaphragmatic activity/ extravasated dose). 


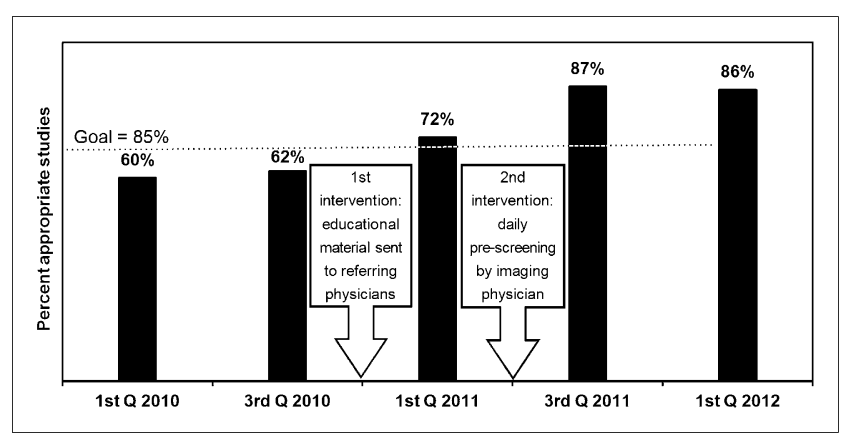

FIGURE 4. Multicycle project graph demonstrating progress of project toward predetermined goal. This example demonstrates appropriateness of myocardial perfusion imaging over time. Once goal was met, monitoring continued to ensure that achievement was maintained. $\mathrm{Q}=$ quarter.

over several years, the graph can show the improvements realized by the incremental interventions (Fig. 4).

To find potential interventions, the team conducts a critical examination of results that fail to meet the criteria. The team drills down to look for possible causes for the failures. Patterns, differences, or aberrations identified may help uncover underlying process deficiencies. The team should list as many system factors as possible rather than focusing on specific people.

At this phase of the inquiry, the goal is to use knowledge and expertise to investigate all possible causes, not to determine all the answers.

\section{Step 7: Share the Results of the Data with Team Members}

On completion of the data collection and analysis phase of the process, a meeting to share the results is extremely important. The meeting should include all staff relevant to the problem and the factors that may cause the problem. The meeting may include interpreting physicians and technologists as well as nurses and administrative staff, as appropriate for the project. The team may invite staff from other departments, if their input would help. If possible, inclusion of management during all or part of the meeting may help expedite key decisions.

The purpose of this meeting is to review the findings of the in-depth analysis and to suggest actions for improvement. The team determines what the department can do differently or better. For a large or complex problem, the team may need several meetings, with additional interval data analysis, to find potential solutions.

\section{Step 8: Select an Action Plan}

Now that the team has collected, analyzed, and discussed the data, the team must prioritize the most promising solutions. The goal is to find the interventions that can lead to systematic change and sustainable improvements.

The selected intervention must be practical and must balance the potential benefits versus the costs. Some seemingly great options may be unusable because of a lack of resources. To make everything perfect is ideal but may be cost-prohibitive. Management must select a course of action and then provide the resources needed to implement the change.

\section{Step 9: Implement the Changes}

Now the staff must implement the chosen changes. The method of implementation must cause the least possible disruption, and the staff must have the time and support necessary to put the change into operation.

The quality improvement team may pilot the solution on a small scale to determine whether the intervention is practical and effective. Test trials of proposed changes may be especially helpful when there are 2 solutions from which to choose.

Everyone, including management, must understand the change and be committed to the outcome. Therefore, the implementation strategy must include time to teach or explain the new procedure and prepare for the transition. Change can be difficult and changing habits takes time.

\section{Step 10: Recollect Data and Analyze}

After sufficient time to allow the selected change to become established, the team must recollect and analyze data by circling back to step 5 of the cycle. Usually, the data are recollected using the same data tools as in the previous cycle. The purpose of recollecting the data is to see whether the change created an improvement. Subsequently, the team must select a goal for the next measurement cycle.

In the earlier example involving the accuracy of myocardial perfusion reports, the initial goal set by the laboratory was $90 \%$ compliance. However, after the first measurement period the results demonstrated only $60 \%$ compliance. At this point, the laboratory might choose a goal of $80 \%$ as an intermediate step toward the ultimate goal of $90 \%$ compliance.

The team repeats the quality improvement process using the steps in the P-D-S-A cycle until the facility reaches its goal. However, simply reaching the goal once is not enough. The team must monitor at selected intervals to ensure that the facility sustains the improvement.

Table 1 shows a sample project using the 10-step quality improvement process.

\section{TYPES OF QUALITY IMPROVEMENT ACTIVITIES}

Quality improvement should involve all aspects of laboratory practice. To help facilities address the different aspects, the Intersocietal Accreditation Commission divides quality improvement into 3 areas: administrative quality, technical quality, and interpretive quality (12).

An administrative-quality review refers to activities designed to assess and improve the laboratory's operation or organizational process. Projects regarding patient wait times, patient satisfaction, completeness of patient demographics, and report turnaround address administrative aspects of the practice.

For example, report turnaround is the time from completion of the imaging study until the facility transmits the 


\begin{tabular}{|c|c|c|}
\hline Stage & Step & Description \\
\hline \multirow[t]{6}{*}{ Plan } & Identify problem & $\begin{array}{l}\text { Nuclear cardiology laboratory that performs exercise and pharmacologic stress myocardial perfusion } \\
\text { imaging notices that many patients arrive for appointment carrying large cup of coffee from new } \\
\text { shop next door. It would be easy to assume that presence of shop is cause of problem. However, } \\
\text { practice decides to investigate if other factors could be involved. }\end{array}$ \\
\hline & Gather information & $\begin{array}{l}\text { Laboratory gathers information about its current procedures, looking at patient preparation form, } \\
\text { scheduling process, and patient confirmation process. }\end{array}$ \\
\hline & $\begin{array}{l}\text { State goal and } \\
\text { set targets }\end{array}$ & $\begin{array}{l}\text { Laboratory sets goal of improving patient compliance with caffeine restriction before myocardial } \\
\text { perfusion imaging. }\end{array}$ \\
\hline & & Laboratory wants $90 \%$ of patients to be in compliance with patient preparation instructions. \\
\hline & $\begin{array}{l}\text { Design data } \\
\text { collection strategy }\end{array}$ & $\begin{array}{l}\text { Laboratory creates simple form to record patient name, whether patient consumed caffeine within } \\
18 \mathrm{~h} \text { of test, staff member who scheduled patient, date patient was scheduled, referring } \\
\text { office and its staff member who scheduled patient, staff member who confirmed } \\
\text { appointment before test, date appointment was confirmed, and whether preparation instructions } \\
\text { were personally discussed with patient. }\end{array}$ \\
\hline & & Laboratory decides, for $2 \mathrm{wk}$, to survey every patient scheduled for myocardial perfusion imaging. \\
\hline \multirow[t]{2}{*}{ Do } & Collect data & For 2 wk, laboratory surveys every patient scheduled for myocardial perfusion imaging. \\
\hline & Analyze data & $\begin{array}{l}\text { Laboratory compiles data into spreadsheet and highlights data associated with patients who } \\
\text { consumed caffeine or for other reasons were inadequately prepared for examination. } \\
\text { Percentage of patients inadequately prepared is calculated. }\end{array}$ \\
\hline \multirow[t]{3}{*}{ Study } & Share results & Laboratory schedules staff meeting. \\
\hline & Select action plan & $\begin{array}{l}\text { After examining data, laboratory realizes that most caffeine-consuming patients came from } \\
\text { one referring office. In addition, many of those patients were not personally reached by staff } \\
\text { member responsible for confirming test. }\end{array}$ \\
\hline & & $\begin{array}{l}\text { Plan is formulated to educate culprit referring office and to revise patient instruction form to } \\
\text { highlight importance of caffeine restriction. }\end{array}$ \\
\hline \multirow[t]{3}{*}{ Act } & Implement change & $\begin{array}{l}\text { Laboratory does in-service training with all staff and conducts lunch-and-learn session with } \\
\text { culprit office to emphasize importance of appropriate patient preparation and consequences of } \\
\text { inappropriate preparation. Revised patient preparation form is provided to culprit office. }\end{array}$ \\
\hline & Repeat & $\begin{array}{l}\text { Laboratory then repeats data collection for } 2 \text { wk and notices decrease in number of coffee-drinking } \\
\text { arrivals who were scheduled from culprit office. }\end{array}$ \\
\hline & & $\begin{array}{l}\text { Laboratory decides to conduct lunch-and-learn sessions and provide new form to all referring } \\
\text { physician offices. }\end{array}$ \\
\hline
\end{tabular}

final report to the referring physician. Published guidelines state that final reports must be sent to the referring physician within $2 \mathrm{~d}$ of study completion, usually 2 business days. Variables a facility can measure include the type of study, which physician interpreted the study, the time of day that the acquisition ended, the processing time, the interpretation time, the dictation time, the transcription time, the physician sign-off time, and the report transmission time.

Some projects seek to improve the technical quality of images or procedures the facility performs. Examples include assessment and improvement of image quality, of region-ofinterest placement, and of patient preparation adequacy.

One technical factor that affects the diagnostic accuracy of many nuclear medicine examinations is whether the patient is physiologically ready for the procedure. This preparation may include such factors as the giving or withholding of specific medications or foods, proper hydration, or assessment of the patient's serum glucose level. If a facility has noticed problems with its oncologic PET/CT studies, it could look for improvement opportunities by comparing the adequacy of patient preparation with scan quality. The facility could study a range of potential variables, including whether the patient had food within $6 \mathrm{~h}$; liquids, except water, within $6 \mathrm{~h}$; parenteral nutrition within $4 \mathrm{~h}$; intravenous dextrose within $4 \mathrm{~h}$; metformin within $12 \mathrm{~h}$; or a serum glucose level greater than $150 \mathrm{mg} / \mathrm{dL}$. The facility might select the study factors based on the facility protocol, national guidelines, or a review of the medical literature.

The last type of quality measure, interpretive, looks at actions to improve physician performance regarding the quality of medical practice. Examples of potential projects are those involving improvement in report accuracy, correlation of interpretation with other diagnostic studies or pathology reports, intraobserver agreement, and interobserver agreement.

For example, interobserver variability is a measurement of the degree to which 2 or more independent observers report the same observed values after measuring the same events (13). In nuclear medicine, interobserver variability is also known as peer review-the degree to which 2 or more interpreting physicians report the same findings when interpreting the same patient study without knowledge of the other physician's interpretation. For example, bone scan interpretation by 2 physicians may be compared to assess agreement on the number, location, and intensity of lesions; other pertinent positive and negative findings; and the final impression. 
The Intersocietal Accreditation Commission Nuclear/PET Web site, www.intersocietal.org/nuclear, has tools for many of the quality improvement activities discussed here.

\section{QUALITY IMPROVEMENT MEETINGS}

The importance of quality improvement meetings cannot be overemphasized. Quality improvement is everyone's responsibility, and because every member of the staff sees the practice from a different perspective, management needs input from all staff to identify the best ways to improve the practice. In addition, involving all stakeholders helps to ensure buy-in for the change and the results. Therefore, the meetings should involve all parties who might have knowledge about factors affecting the project.

Also, everyone must meet periodically to share in the results and the successes. In a large department, a gathering of the full staff might occur only once or twice a year, whereas in a smaller department it might occur every 1-3 mo. Sharing the information helps everyone feel part of the improvement process and gets everyone thinking about more ideas for projects.

Management must document the key points presented and discussed at the meetings. Minutes are useful to provide evidence of quality improvement activities for accreditation bodies, payers, and government agencies.

Conducting a meeting with all involved staff, reviewing the results of quality measures, documenting findings, and formalizing a plan for improvement increases the likelihood of lasting change and an outcome of significant improvement.

\section{CONCLUSION}

The goal of quality improvement is just as it sounds- to improve the quality of what the department does. Quality improvement activities in nuclear medicine should focus on patient safety, the accuracy of results, the patient's experience, and the efficiency of processes across all aspects of the practice.

When monitoring confirms compliance with goals, the facility does not need an improvement project for that area. The facility should select a new measure to help identify a new project for the next cycle. However, if the facility finds a performance gap, then the facility should use the quality improvement cycle to analyze data and identify possible changes to close the gap. After the intervention is in place, the quality improvement team remeasures to confirm that the change resulted in improvement.

Many laboratories claim, "I don't have time to do quality improvement." The truth is they must make time. The cost of poor quality and wasted resources is great.

\section{APPENDIX A}

\section{Example of P-D-S-A Problem-Solving Model: Quality of Myocardial Perfusion Imaging}

The P-D-S-A model can be used to assess and improve the quality of myocardial perfusion images by evaluating rotating cine planar images and scoring the images on the basis of parameters established by the American Society of Nuclear Cardiology.

\section{Plan}

Rotating cine images can be evaluated in terms of patient motion, breast attenuation, diaphragmatic attenuation, extracardiac activity, liver count density (hot liver), study count statistics, gating accuracy, and overall quality (8). After deciding on the study variables, the method of assessing each variable is determined. For instance, patient motion can be graded as none, mild, moderate, or severe, and gating can be assessed by the presence or absence of flickering or flashing as the cine rotates.

The next step is to determine the level of each variable that is acceptable or unacceptable and thus counted. Again using patient motion as an example, no patient motion or mild motion may be considered acceptable. However, moderate or severe patient motion is unacceptable.

At this point, a threshold is set for each variable to predict a starting point for acceptable levels. For instance, a laboratory may decide to set a threshold for patient motion of no more than $10 \%$, thereby determining that initially the laboratory will tolerate significant patient motion in 1 of 10 studies.

Patient cines may be randomly selected over a specific period, or consecutive patients can be evaluated. A minimum of 30-55 patient cines should be evaluated (Fig. 1A).

\section{Do}

Once the variables have been selected and thresholds set, it is time to collect the data. After the data have been collected, they can be analyzed by using simple percentages for each variable and determining whether the threshold was met. For instance, by adding the number of cine images that demonstrate moderate or severe patient motion, dividing by the total sample, and multiplying by $100 \%$, one can determine the percentage of studies with patient motion. This number is then compared against the threshold value to determine whether the threshold has been exceeded.

\section{Study}

After the data are analyzed, the team examines the data and drills down to find ways to improve. For example, if the results demonstrate that the laboratory has a greater number of low-count studies than expected, the data can be further

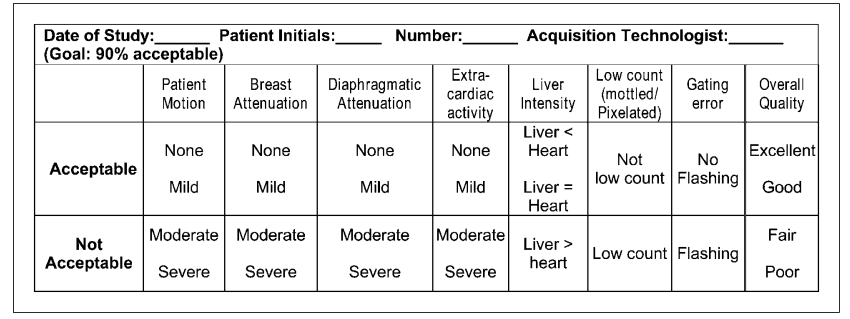

FIGURE 1A. Sample data collection tool to assess improvement in quality of myocardial perfusion images. 
evaluated to see if the low-count studies are the result of patients being obese or perhaps dose infiltration. If the low-count studies are the result of patients being obese, the laboratory may decide to extend imaging time, use weight-based dosing, or implement a 2-d protocol for obese patients.

\section{Act}

Finally, the results and solutions are shared with all staff. The interventions are selected and then implemented. Data are recollected to gauge the effect of the intervention. If the new results show fewer low-count studies because of the chosen changes, then these changes are made a standard operating procedure. If the threshold is not met, a need for more action is indicated. The laboratory must conduct further analyses to look for other potential interventions. The laboratory may find that intermediate goals are needed on the way to reaching the ultimate target. As long as further change is needed, the P-D-S-A cycle is repeated.

\section{ACKNOWLEDGMENT}

No potential conflict of interest relevant to this article was reported.

\section{REFERENCES}

1. Thompson-Hunt K, Mars DC, Mehlberg LM. Patient Care and Quality Improvement. In: Christian P, Waterstram-Rich K, eds. Nuclear Medicine and PET/CT
Technology and Techniques. 6th ed. St. Louis, MO: Mosby Elsevier; 2007:253265.

2. Patient safety-quality improvement. Duke University Web site. Available at: http://patientsafetyed.duhs.duke.edu/module_a/introduction/introduction.html. Accessed September 25, 2012

3. Cochran C. The Continual Improvement Process: From Strategy to the Bottom Line. Chico, CA: Patton Professional; 2003:71-91.

4. Ogrinc GS, Headrick LA, Moore SM, Barton AJ, Dolansky MA, Madigosky WS. Fundamentals of Health Care Improvement: A Guide to Improving Your Patients' Care. 2nd ed. Oakbrook Terrace, IL: The Joint Commission and the Institute for Healthcare Improvement; 2010:139-141.

5. Imaging guidelines for nuclear cardiology procedures. American Society of Nuclear Cardiology Web site. Available at: http://www.asnc.org/content_184. $\mathrm{cfm}$ ?navID=73. Accessed September 25, 2012

6. Papp J. Quality Management in the Imaging Sciences. 3rd ed. St. Louis, MO: Mosby Elsevier: 2006:17-30.

7. Hendel RC, Cerqueira M, Douglas PS, et al. A multicenter assessment of the use of single-photon emission computed tomography myocardial perfusion imaging with appropriateness criteria. J Am Coll Cardiol. 2010;55:156-162.

8. The importance of quality sample size. Unite for Sight Web site. Available at: http://www.uniteforsight.org/global-health-university/importance-of-qualitysample-size. Accessed September 25, 2012.

9. Calculating sample size. Health Resources and Services Administration Web site. Available at: http://bphc.hrsa.gov/policiesregulations/performancemeasures/ patientsurvey/calculating.html. Accessed September 25, 2012

10. Acceptance sampling procedures under certain specific licenses. Fed Regist. 2012;39:22130. Codified at 10 CFR $\$ 32.110$.

11. Carey RG, Lloyd RC. Measuring Quality Improvement in Healthcare: A Guide to Statistical Process Control Applications. New York, NY: Quality Resources; 1995:32-35.

12. The IAC standards and guidelines for nuclear/PET accreditation. Intersocietal Accreditation Commission Web site. Available at: http://www.intersocietal.org/ nuclear/standards/IACNuclearPETStandards2012.pdf. Accessed September 25, 2012.

13. Definition of observer variation. MedicineNet.com Web site. Available at: http:// www.medterms.com/script/main/art.asp?articlekey $=8056$. Accessed September $25,2012$. 